\title{
Thermal Stress Measurement of Tungsten fiber Reinforced Titanium by X-ray Diffraction
}

\author{
Masayuki Nishida* Masashi Haneoka** Ryoji Hirai** Tatsuya Matsue*** and \\ Takao Hanabusa**** \\ * Depatment of Mechanical Engineering, Kobe City College of Technology \\ 8-3 Gakuenhigashi-machi, nishi-ku, Kobe, Japan \\ E-mail:nishida@kobe-kosen.ac.jp \\ ** Student of Advanced Course, Kobe City College of Technology \\ 8-3 Gakuenhigashi-machi, nishi-ku, Kobe, Japan \\ *** Department of Environmental Materials Engineering, Niihama National College of Technology \\ 7-1, Yakumo-cho, Niihama, Ehime, Japan \\ **** The University of Tokushima
}

2-1, Minamijosanjima-cho, Tokushima, Tokushima, Japan

\begin{abstract}
Titanium is one of the useful materials in industrial fields because of the good properties of light weight, high temperature resistance and so on. In this study, the tungsten fiber reinforced titanium composite (W/Ti) was produced by the spot welding method. This manufacturing method used only a simple spot welding system, and it did not need a vacuum chamber and a high temperature furnace such as existing common methods. The arranged tungsten fibers were held between titanium plates (thickness $0.5 \mathrm{~mm}$ ) and fixed by the spot welding. Therefore, this W/Ti composite produced by the spot welding did not joint in the whole position between the tungsten fiber and the titanium matrix because of the partial welding in spot welding points. The coverage, a rate of welding area to the whole plate area, became in $100 \%$ for the sample in this study, because it should make up for the partial welding by this method. X-ray stress measurement is the most useful non-distractive method for estimating residual stresses on material surface. It is inferred the W/Ti sample included thermal stresses generated from the thermal expansion coefficient between the titanium matrix and the tungsten fiber. These thermal stresses of the W/Ti composite were measured by $\mathrm{x}$-ray stress measurement. Furthermore, the alteration of thermal residual stress under the thermal cycling was measured by the in-situ $\mathrm{x}$-ray stress measurement technique. These results were discussed from the viewpoint of the thermal expansion coefficient between fiber and matrix.
\end{abstract}

Key words: W/Ti composite, Spot welding, X-ray stress measurement, Thermal stresses, In-situ stress measurement

\section{Introduction}

Fiber-reinforced materials have found considerable application in the improvement of the performance characteristics of engineering materials. Commonly, there is a large difference in thermal expansion coefficients between the matrix and the fiber, which causes thermal stress development in a composite system ${ }^{(1,2)}$. The effective method has been made on analyzing the thermal stress on the surface of composite materials by the $\mathrm{x}$-ray stress measurement technique ${ }^{(2,3)}$. This method is essentially based on the $\sin ^{2} \psi$ method ${ }^{(4)}$.

In this study, thermal residual stresses in the continuous tungsten fiber reinforced 
titanium matrix composite (W/Ti composite) were examined by the x-ray stress measurement method. This W/Ti composite was produced by the simply spot welding method. Furthermore, stress alterations during high temperature cycling were estimated in the W/Ti composite by the combination of x-ray diffraction and a high temperature vacuum furnace system ${ }^{(3)}$. Temperature is changed from $15^{\circ} \mathrm{C}$ to $700^{\circ} \mathrm{C}$ periodically, and thermal stresses in the tungsten fiber and the titanium matrix were measured at each temperature.

\section{Preparation of Fiber Reinforced Material}

A fiber reinforced metal matrix composite was produced for the present investigation. In this composite, $99.9 \%$ purity tungsten fiber with $100 \mu \mathrm{m}$ diameter and $99.9 \%$ purity titanium were used for the fiber phase and the matrix phase, respectively.

In this study, the tungsten fiber reinforced titanium composite (W/Ti) was produced by the spot welding method. This manufacturing method used only a simple spot welding system, and it did not need a vacuum chamber and a high temperature furnace such as existing common methods. The arranged tungsten fibers were held between titanium plates (thickness $0.5 \mathrm{~mm}$ ) and fixed by the spot welding. Therefore, this W/Ti composite produced by the spot welding did not joint in the whole position between the tungsten fiber and the titanium matrix because of the partial welding in spot welding points. The coverage, a rate of welding area to the whole plate area, became in $100 \%$ for the sample in this study, because it should make up for the partial welding by this method.

Fig. 1(a) shows the schematic diagram of the manufacturing method for the W/Ti composite by the spot welding. The tungsten fiber winded on the titanium plate (thickness $0.5 \mathrm{~mm}$ ) was slipped in between the other titanium plates. These three plates whose the center plate was winded the continuous tungsten fiber were welded by the spot welding system. This W/Ti composite produced by the spot welding did not joint in the whole position between the tungsten fiber and the titanium matrix because of the partial welding in spot welding points. Therefore, the spot welding must be overlapped with the next welding point. Fig. 1(b) shows the spot welding positions which define the coverage for W/Ti composite. The coverage, a rate of welding area to the whole plate area, became in $100 \%$ for this sample in Fig. 1(b).

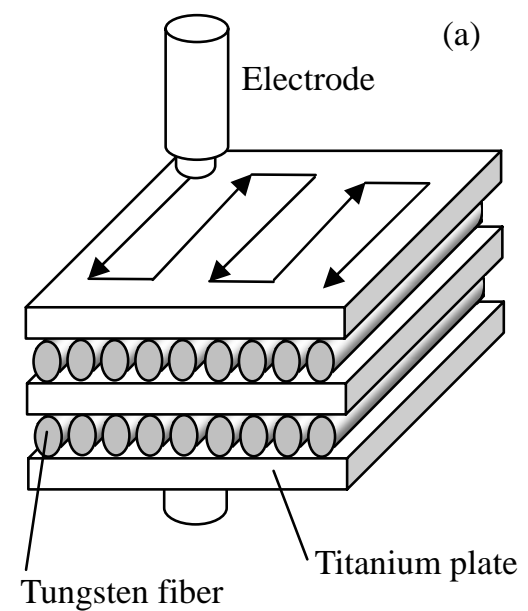

Fig.1 Schematic diagram of: (a) the manufacturing procedure by the spot welding method, (b) the spot welding positions which define the coverage for W/Ti composite. 
Fig.2 shows photographs of the W/Ti composite. Fig.2 (a) shows a lot of indentations by the spot welding were observed on the sample surface. Fig.2 (b) is the crophotograph of the cross section of W/Ti composite. The distance between the tungsten fibers is about $0.5 \mathrm{~mm}$. Fig.2 (c) is the specimen surface. This surface was polished by \#1500 emery paper. The conditions of the spot welding were shown in Table 1.

(a)
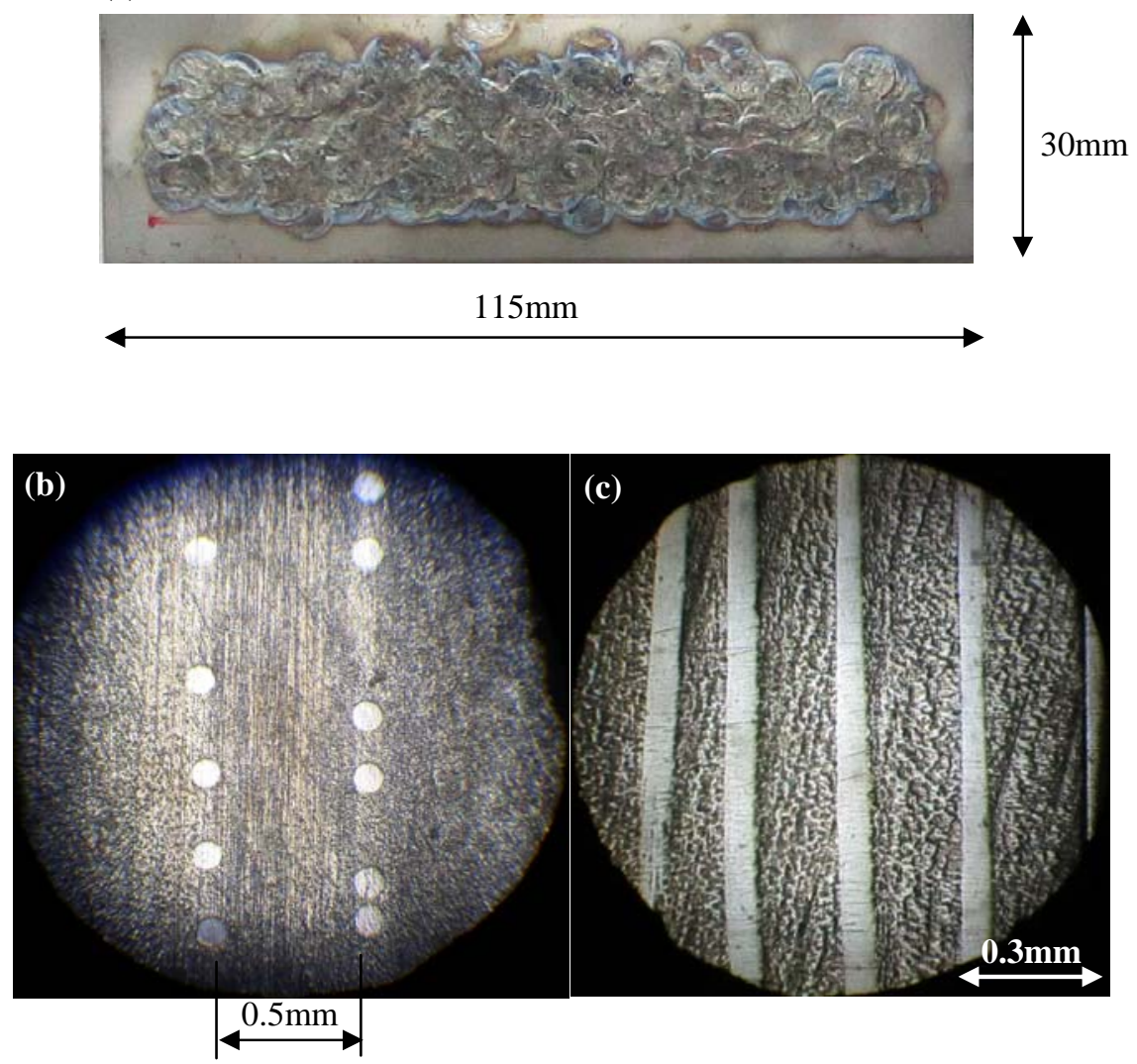

Fig.2 Photographs of the W/Ti composite; (a) a lot of indentations by the spot welding were observed on the sample surface, (b) a microphotograph of the cross section of W/Ti composite, (c) the specimen surface polished by \#1500 emery paper.

Table 1 Conditions of spot welding.

\begin{tabular}{c|c}
\hline Welding voltage \& current & $200 \mathrm{~V}, 7.8 \mathrm{~A}$ \\
\hline Welding pressure & $1.9 \mathrm{kN}$ \\
\hline Holding time & $200 \mathrm{msec}$. \\
\hline Diameter of electrode & $11 \mathrm{~mm}$ \\
\hline
\end{tabular}

After the spot welding, the surface of the specimen was polished with \#1500 emery paper in order to strip off the indentations of the spot welding. Therefore, the surface of W/Ti sample has residual stresses caused by emery polishing. These residual stresses were estimated by the x-ray stress measurement. The final dimension of the sample for the in-situ thermal residual stress measurement was $18 \mathrm{~mm} \times 15 \mathrm{~mm}$, thickness $1.5 \mathrm{~mm}$. The volume fraction of the tungsten fiber is about $5 \%$ in this sample.

\section{Theory of X-ray Stress Measurement}

The long-fiber reinforced composite considered in this investigation is schematically 
shown in Fig. 3. The $x_{1}$ and $x_{2}$ axes are parallel to and the $x_{3}$ axis is normal to the specimen surface, with the $x_{1}$ axis being defined as parallel to the fiber direction. The normal lattice strain $\boldsymbol{\varepsilon}_{\phi \psi}$ of a particular $h k l$ plane defined by the angles $\phi$ and $\psi$ is also shown in this figure.

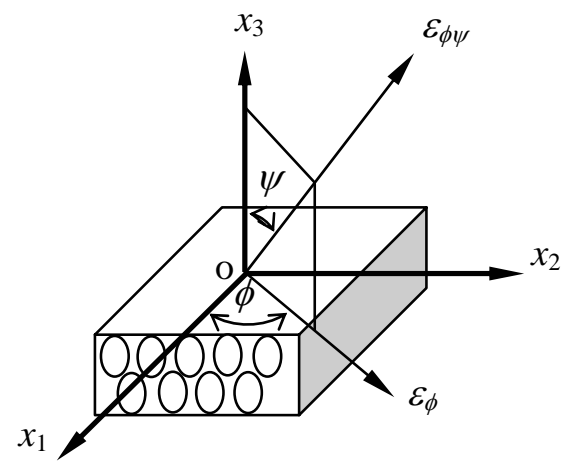

Fig.3 Coordinate system and definition of the $\psi$ and $\phi$ angles.

According to the theory of isotropic elasticity, the strain $\boldsymbol{\varepsilon}_{\phi \psi}$ is given by the following equation:

$$
\begin{aligned}
& \varepsilon_{\phi \psi}=\frac{1+v}{E}\left(\sigma_{11} \cos ^{2} \phi+\sigma_{12} \sin 2 \phi+\sigma_{22} \sin ^{2} \phi-\sigma_{33}\right) \sin ^{2} \psi+\frac{1+v}{E} \sigma_{33} \\
& -\frac{v}{E}\left(\sigma_{11}+\sigma_{22}+\sigma_{33}\right)+\frac{1+v}{E}\left(\sigma_{23} \sin \phi+\sigma_{31} \cos \phi\right) \sin 2 \psi,
\end{aligned}
$$

where $E$ is Young's modulus, $v$ is Poisson's ratio. To consider the longitudinal stress $\sigma_{11}$ parallel to the fibers, we define the angle $\phi$ equals zero. It was previously reported that both the stresses parallel normal to the fiber are principal stresses and no shearing stress exists ${ }^{(2)}$. Therefore, the Eq. (1) is transformed into Eq. (2) for $\phi=0^{\circ}$.

$$
\varepsilon_{\phi=0, \psi}=\frac{1+v}{E}\left(\sigma_{1}-\sigma_{3}\right) \sin ^{2} \psi+\frac{1+v}{E} \sigma_{33}-\frac{v}{E}\left(\sigma_{1}+\sigma_{2}+\sigma_{33}\right) .
$$

If the plane stress condition is maintained in the surface layer, we can assume the stress $\sigma_{3}=0$ and Eq.(2) change to following equation.

$$
\varepsilon_{\psi}=\frac{1+v}{E} \sigma_{1} \sin ^{2} \psi-\frac{v}{E}\left(\sigma_{1}+\sigma_{2}\right)
$$

From this equation, it means that the relationship of $\varepsilon_{\psi}$ vs. $\sin ^{2} \psi$ is linear, and the longitudinal stress $\sigma_{1}$ is determined from the slope of the $\boldsymbol{\varepsilon}_{\psi}$ - $\sin ^{2} \psi$ diagram. Therefore, the $\sin ^{2} \psi$ method can be used for the estimation of the stress condition in surface region.

Furthermore, when $\boldsymbol{\varepsilon}_{\psi}$ is calculated from the diffraction angle $2 \theta_{\psi}$,

$$
\varepsilon_{\psi}=\frac{d_{\psi}-d_{0}}{d_{0}}=-\left(\theta_{\psi}-\theta_{0}\right) \cot \theta_{0} \cdot \frac{\pi}{180},
$$

where $\theta_{0}$ (degrees) is the diffraction angle of the stress free state. The $2 \theta-\sin ^{2} \psi$ diagram can be obtained by measuring $2 \theta$ at several values of $\psi$ angles. By substituting Eq.(4) into Eq.(3), we obtain the following.

$$
\sigma_{1}=-\frac{E}{2(1+v)} \cot \theta_{0} \frac{\partial\left(2 \theta_{\psi}\right)}{\partial\left(\sin ^{2} \psi\right)} \cdot \frac{\pi}{180}
$$

The gradient of the regression line in this $2 \theta-\sin ^{2} \psi$ diagram is equal to the term $\partial(2 \theta) / \partial\left(\sin ^{2} \psi\right)$ in Eq. (5). 


\section{In-situ Thermal Stress Measurement}

In this study, stress alterations caused from high temperature cycling was measured by the in-situ $\mathrm{x}$-ray stress measurement method. The high temperature vacuum furnace was mounted on the diffractometer system. The sample of the W/Ti composite was installed on the sample holder in the vacuum furnace. In this measurement, the thermal residual stresses $\sigma_{1}$ parallel to the longitudinal direction, see in Fig.1, of the tungsten fiber were measured in the tungsten fiber and titanium matrix, respectively.

Fig. 4 shows the schematic diagram of temperature vs. time program for in-situ stress measurement of W/Ti composite. The temperature program was controlled by PID temperature controller automatically. Temperature was repeated three times from $15^{\circ} \mathrm{C}$ to $700^{\circ} \mathrm{C}$ in case of the tungsten fiber. On the other hand, the temperature change was from $15^{\circ} \mathrm{C}$ to $550^{\circ} \mathrm{C}$ in case of the titanium matrix, because the titanium oxide was generated on the sample surface over $600^{\circ} \mathrm{C}$.
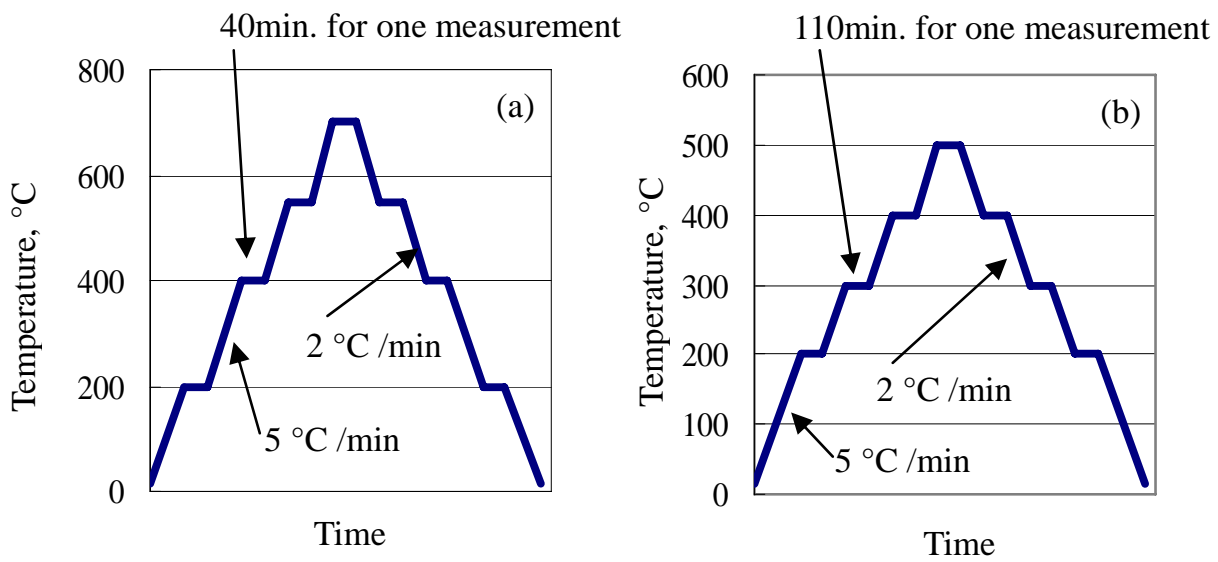

Fig.4 Temperature programs of in-situ stress measurement for; (a) tungsten fiber, (b) titanium matrix in W/Ti composite.

The rate of temperature change is about $5^{\circ} \mathrm{C} / \mathrm{min}$ in heat up stage and $2^{\circ} \mathrm{C} / \mathrm{min}$ in cool down stage. When the temperature came to a target position, it was holding for about 10 minutes in order to stabilizing, and after that the stress measurement started in every case. The measuring time for $\mathrm{x}$-ray stress measurement in each temperature is about 40 minutes for the tungsten fiber and 110 minutes for the titanium matrix.

Table 2 conditions of stress measurement.

\begin{tabular}{c|c}
\hline Characteristic x-rays & $\mathrm{CuK} \alpha$ \\
\hline X-ray optics & Parallel beam \\
\hline Tube voltage & $40 \mathrm{kV}$ \\
\hline Tube current & $25 \mathrm{~mA}$ \\
\hline $\sin ^{2} \psi$ & $1.0,0.5$ \\
\hline hkl plane \& Diffraction angle & W 321, $2 \theta=131.1^{\circ}$ \\
$2 \theta$ step angle \& fixed time & Ti $213,2 \theta=139.3^{\circ}$ \\
\hline Filter & Ti: $0.1^{\circ}, 40 \mathrm{sec}$. \\
\hline Irradiated area & Nikkel \\
\hline Peak deciding method & $10 \times 5$ mm \\
\hline
\end{tabular}


Table 2 shows the conditions of the in-situ x-ray stress measurement. In this measure, only two points of the $\psi$ angle $\left(0^{\circ}\right.$ and $\left.45^{\circ}\right)$ were employed, because the sash bar of the furnace window was cut the $\mathrm{x}$-ray beam.

The intensity of diffraction beam was very weak because the diffraction beam passed through the window of the vacuum furnace. Especially, the diffraction profile from the titanium matrix included the high level back ground because the fluorescence $x$-ray was generated in the titanium sample. Therefore, we had to keep long fixed times in each $2 \theta$ step in order to the accurate and clear measurement. The fixed time is 40 seconds for the diffraction from the tungsten fiber and 90 seconds for the titanium matrix.

\section{Results and Discussions}

Fig. 5 shows the x-ray diffraction profile of W/Ti composite. The diffraction peaks of tungsten fiber and titanium matrix were appeared clearly. The marks $\bigcirc$ and $\bigcirc$ in Fig.5 are diffraction peaks from the tungsten fiber and the titanium matrix, respectively. The diffraction peaks of $\mathrm{W} 321,2 \theta=131.1^{\circ}$ and $\mathrm{Ti} 213,2 \theta=139.3^{\circ}$ were employed in this $\mathrm{x}$-ray stress measurement.

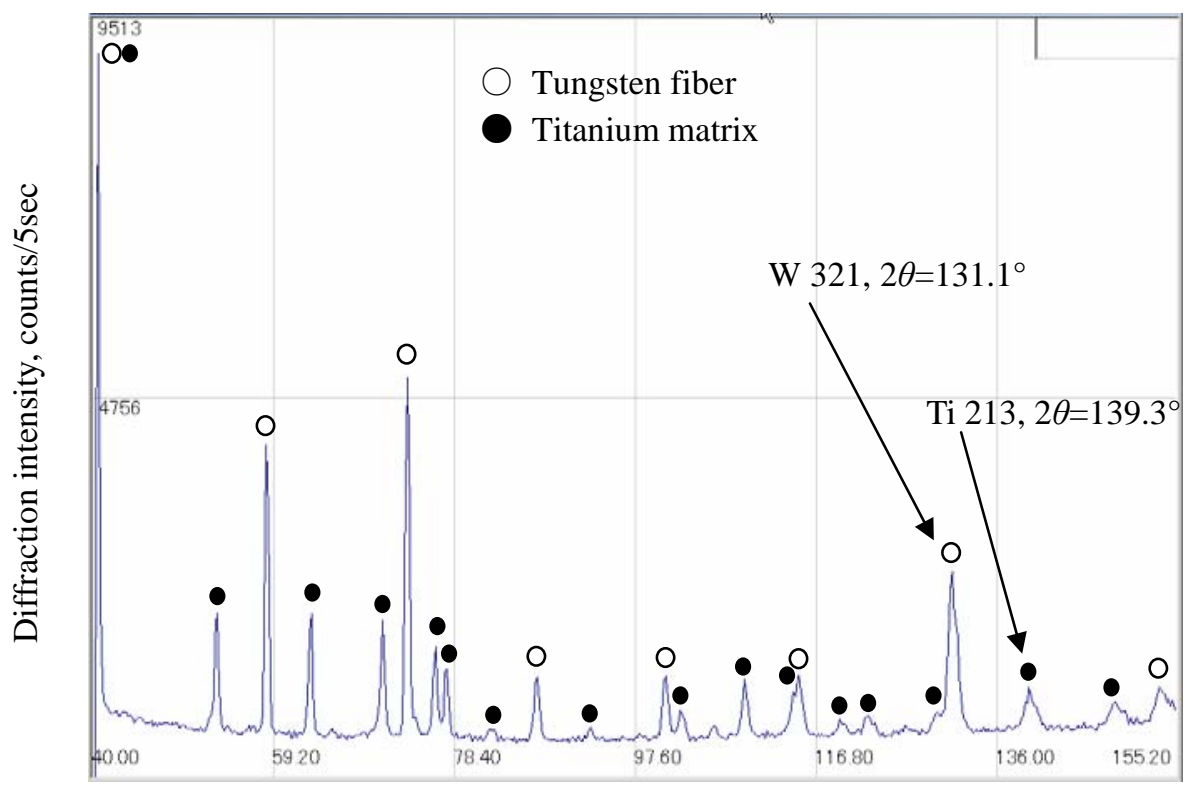

Diffraction angle $2 \theta$, deg.

Fig.5 Diffraction profile of W/Ti composite. The tungsten fiber exposed on the surface of W/Ti composite by \#1500 emery polished.

From Fig.5, the diffraction profile from W 321 overlapped a small titanium peak. However, this peak was ignored for the measurement because of the smallness intensity comparing the tungsten peak. On the other hand, the textile structure was not observed in both of the tungsten fiber and the titanium matrix. Therefore, the peak intensity from the both materials was enough to measure the thermal residual stresses in the every $\psi$ tilt angles.

Fig.6 and Fig.7 show results of the in-situ thermal stress measurement against the longitudinal direction stress $\sigma_{1}$ in the tungsten fiber and the titanium matrix, respectively. The result of the first heat up stage is plotted by the dotted line in red. The blue lines are the result of the thermal cycle stage after the first heat up stage. From these results, the initial residual stress in tungsten fiber is compressive state about -820MPa and titanium matrix is 
also compressive about -290MPa. It is supposed that the both of compressive residual stress states in tungsten fiber and titanium matrix were generated by the emery polishing in the initial treatment for the $\mathrm{W} / \mathrm{Ti}$ composite.

From the result of tungsten fiber in Fig.6, the initial residual stress decreased gradually with the temperature raising under the first heat up stage, and it changed to about $-10 \mathrm{MPa}$ in $550^{\circ} \mathrm{C}$. These thermal stresses are generated from a difference of the thermal expansion between the tungsten fiber and the titanium matrix.

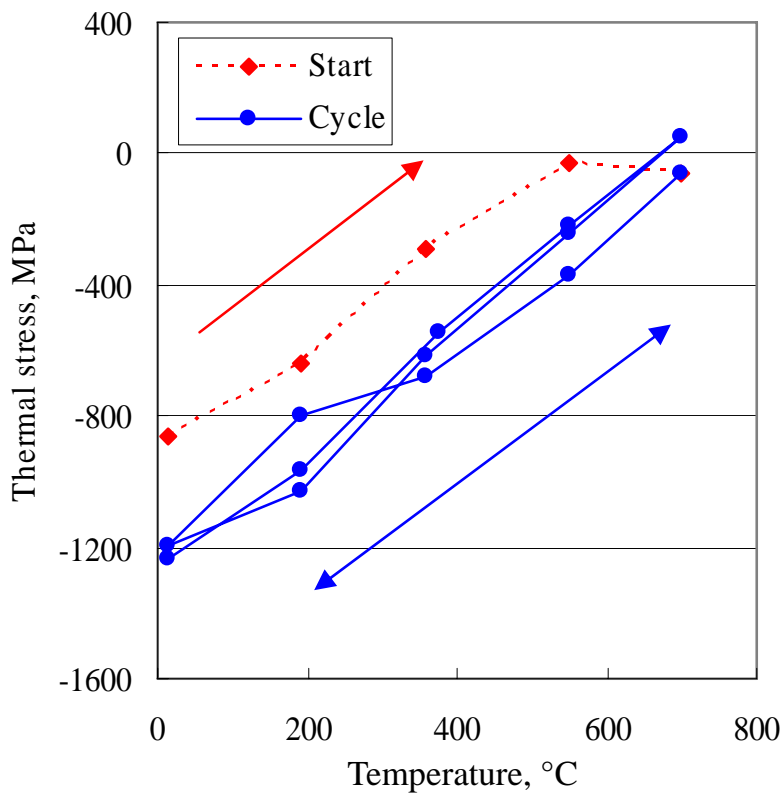

Fig.6 Measurement results of the thermal stress alteration in tungsten fiber under the thermal cycling from $15^{\circ} \mathrm{C}$ to $700^{\circ} \mathrm{C}$. The result of the first heat up stage is plotted the dotted line in red. The blue lines are the result of thermal cycling after the first heat up stage.

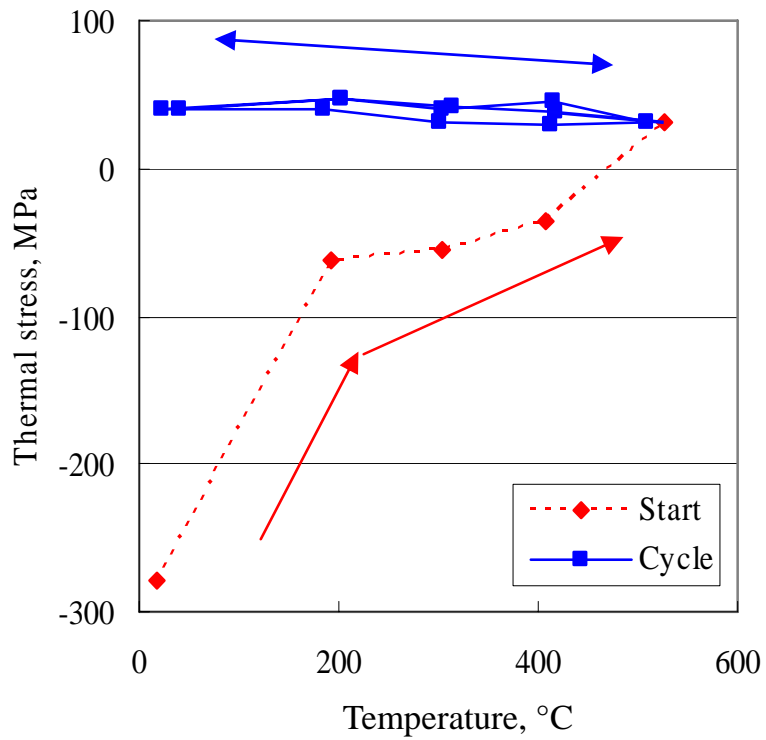

Fig.7 Measurement results of the thermal stress alteration in titanium matrix under the thermal cycling from $15^{\circ} \mathrm{C}$ to $500^{\circ} \mathrm{C}$. The first heat upstage and the other cycling are as well as Fig.6. 
In second cool down stage, thermal stresses increasing from -10MPa to -1200MPa with the temperature dropping. Furthermore, in the next heat up and down stage, thermal residual stresses changed through the almost same route of the second cool down stage.

Fig.7 shows the thermal/residual stress alteration of the titanium matrix. The initial compressive residual stress -290MPa was changed to the tensile state $30 \mathrm{MPa}$ at $500^{\circ} \mathrm{C}$ with the first temperature rising. It is assumed that the initial residual stress in compressive state was caused by the emery polish, and this compressive residual stresses were cancelled in the high temperature. On the other hand, the tensile thermal stresses were generated from the thermal expansion mismatch between the tungsten fiber and the titanium matrix in $500^{\circ} \mathrm{C}$. In second cool down stage, thermal stresses gradually increase from 30MPa to $45 \mathrm{MPa}$ with the temperature dropping. In the next heat cycling stage, thermal residual stresses changed through the almost same route of the second cool down stage.

From Fig.6 and Fig.7, if it is considered the balance of thermal stresses between the tungsten fiber and the titanium matrix, these results may not contradict the stress balance qualitatively, because the thermal stresses of the tungsten fiber and the titanium matrix existed in compressive state and tensile state respectively, except the first heat up stage.

Regarding to these stress alterations in thermal cycling, it is assumed that these phenomena are brought about a difference of thermal expansion between tungsten fiber and titanium matrix.

Fig.8 shows the comparison of the elastic calculation and experimental results in the tungsten fiber and the titanium matrix. In this figure, the continuous lines means the average of experimental results of the thermal cycling after first heat up stage, and the dotted line means the results of simple elastic calculation by the following theoretical equation:

$$
\begin{gathered}
\sigma_{W}=\frac{E_{W} E_{T i} V_{T i}}{E_{W} V_{W}+E_{T i} V_{T i}}\left(\alpha_{W}-\alpha_{T i}\right) \Delta T \\
\sigma_{T i}=\frac{E_{W} E_{T i} V_{W}}{E_{W} V_{W}+E_{T i} V_{T i}}\left(\alpha_{W}-\alpha_{T i}\right) \Delta T,
\end{gathered}
$$

where suffixes mean materials, $E$ is young modulus, $\alpha$ is coefficient thermal expansion, $\Delta T$ is temperature difference and $V$ is volume fraction. Table 3 shows parameter of this elastic calculation. The stress free position was fixed on the maximum temperature position at $700^{\circ} \mathrm{C}$ in the tungsten fiber and at $500^{\circ} \mathrm{C}$ in the titanium matrix for the sake of convenience.

Table 3. Parameters of elastic calculation.

\begin{tabular}{|c|c|c|c|}
\hline & $\begin{array}{c}\text { Young modulus: } \\
E, \mathrm{GPa}\end{array}$ & $\begin{array}{c}\text { T. exp. Coefficient: } \\
\alpha, \times 10^{-6} 1 /{ }^{\circ} \mathrm{C}\end{array}$ & Volume fraction: $V, \%$ \\
\hline Titanium & 106.0 & 8.4 .0 & 95 \\
\hline Tungsten & 417.7 & $4.5 \times 10^{-6}$ & 5 \\
\hline
\end{tabular}

From these results, the calculated thermal stresses of tungsten fiber and titanium matrix exist in the compressive state and the tensile state, respectively. Furthermore, these stresses increase with the temperature change from the high temperature to the room temperature. Therefore, the experimental results and the elastic calculations coincide with qualitatively. However, the experimental stress values were larger than the calculated results in every temperature. Especially, the stress value of tungsten fiber at $15^{\circ} \mathrm{C}$ is $-1200 \mathrm{MP}$ in experimental result. This value is too large for the case of the volume fraction $5 \%$ in the calculated value. If the volume fraction of tungsten fiber is assumed in $0.5 \%$, the calculated stress value becomes -1150MPa which is near the experimental stress value -1200MPa. 


\section{ATEM'11}

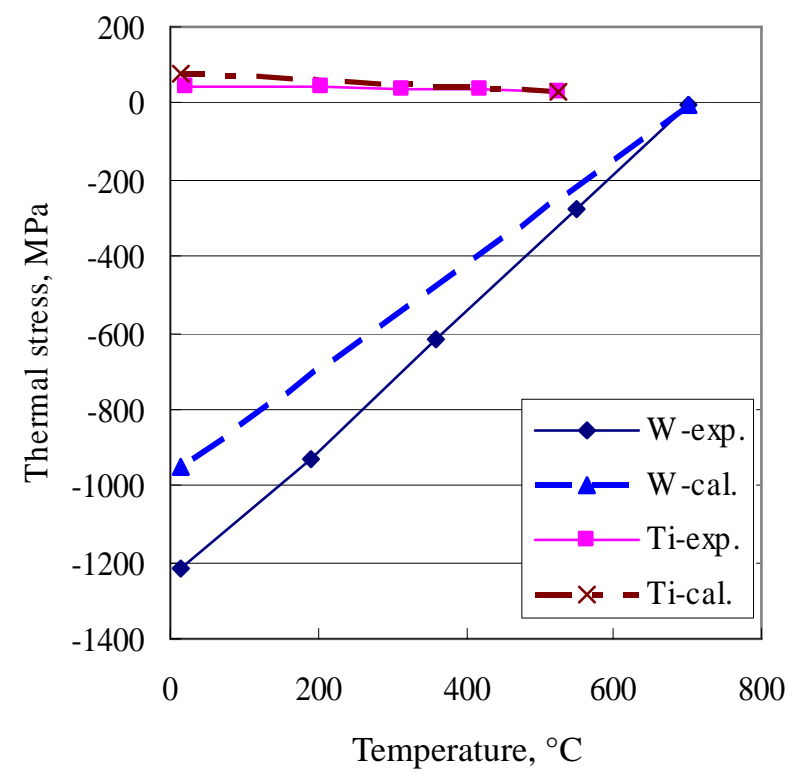

Fig.8 Comparison of results of the elastic calculation and the experimental for the tungsten fiber and the titanium matrix.

The cause of these inconsistencies between both stresses is not found as yet, and this is one of the important problems in feature investigation.

\section{Conclusions}

1. The tungsten fiber reinforced titanium composite was manufactured by the spot welding method.

2. Regarding to the initial residual stress $\sigma_{1}$ in fiber longitudinal direction, the compressive stress existed in the both of the tungsten fiber and titanium matrix.

3. Thermal residual stresses $\sigma_{1}$ in tungsten fiber and titanium matrix were changed to other states depending on temperature changes.

4. The main factor of thermal stress alteration is difference of thermal expansion between tungsten fiber and titanium matrix.

\section{References}

(1) Y. Ikeuchi, T. Matsue and T. Hanabusa, Proc. 5th Int. Conf. on Residual Stresses, Vol. 2(1997), pp. 958-963.

(2) Y.Ikeuchi, K. Iga and T. Hanabusa, J. Soc. Mat. Sci., Japan, Vol 43(1994) 792-798.

(3) M. Nishida, T. Hanabusa, Y. Ikeuchi and N. Minakawa, Mat.-wiss. u. Werkstofftech., Germany, Vol 34(2003)49-55.

(4) The Society of Materials Science Japan ed. Standard Method for X-Ray Stress Measurement, (2005), ISBN 4-901381-27-X, The Society of Materials Science Japan. 